Bangladesh J Otorhinolaryngol 2020; 26(1): 1-4

\title{
Editorial
}

\section{Obstructive Sleep Apnoea and Bangladesh Perspective}

Sleep medicine is a medical superspeciality devoted to the diagnosis and therapy of sleep disturbance and disorders. From the middle of $20^{\text {th }}$ century research has provided increasing knowledge and answered many questions about sleep awake functioning. Disorders and disturbances of sleep are widespread and can have significant consequences for affected individuals as well as economic and other consequences for the society. sleep deprivation has also been a significant factor in dramatic accidents. The first sleep clinic in United states were established in 1970s.

The International Classification of Sleep Disorders (ICSD), the concept of sleep medicine belongs to the second half of the 20 th century. Due to the rapidly increasing knowledge about sleep, including the growth of the research field chronobiology from about 1960 and the discoveries of REM sleep (1952-53) and sleep apnea (first described in the medical literature in 1965), the medical importance of sleep was recognized.

The World Federation of Sleep Research \& Sleep Medicine Societies (WFSRSMS) was established in 1987. The European Sleep Research Society (ESRS) is a member of the WFSRSMS. The Assembly of National Sleep Societies (ANSS), which includes both medical and scientific organizations from 26 countries as of 2007 , is a formal body of the ESRS.The American Academy of Sleep Medicine (AASM), founded in 1978, administered the certification process and sleep medicine examination for doctors.
Elites of Bangladesh having opportunities to get treatment from abroad were availing treatment by surgery or c-pep machines for long time but our common population is not aware of the treatment opportunities for sleep disorders. Above all our repertory physicians and Otolaryngologists were providing limited treatment for sleep apnea. Association of Surgeons for Sleep Apnoea, Bangladesh was established in 2014. Since then we were organizing International conference of Association of Surgeons for Sleep Apnoea, Bangladesh, with Scientific sessions, live surgery workshop and Rallies.

Sleep apnea is a disorder in which breathing is repeatedly interrupted during sleep. The word "apnea" literally means "without breath". An apnea is defined as a complete cessation of breathing that lasts 10 seconds or greater. There are two principal types of sleep apnea, "obstructive sleep apnea (OSA)," and "central sleep apnea" (CSA). Complex sleep apnea is a combination of obstructive sleep apnea and central sleep apnea. Obstructive sleep apnea is an increasingly wellrecognized disease characterized by periodic collapse of the upper airway during sleep. This leads to either complete or partial obstruction of the airway, resulting in apneas, hypopneas, or both. This disorder causes daytime somnolence, neurocognitive defects, and depression. It affects almost every system in the body, resulting in an increased incidence of hypertension, cardiovascular disease, stroke, pulmonary hypertension, cardiac arrhythmias, and altered immune function. It also increases 
the risk of having an accident, presumably as a result of associated somnolence. Like obstructive sleep apnea, central sleep apnea is more common in males and people over the age of 65 . However, unlike obstructive sleep apnea, central sleep apnea is often associated with serious illness, such as heart disease, stroke, neurological disease, or spinal or brainstem injury.

By far the most common is obstructive sleep apnea, comprising 90 to $96 \%$ of apneas diagnosed on overnight sleep study (polysomnography). OSA results from repetitive collapse of the upper airway. Central sleep apnea is due to decreased output from ventillatory control centres in the brain. In clinical practice, the most frequently used measurement of OSA is the Apnoea Hypopnea Index (AHI) derived from an overnight sleep study. It is the number of apneas (complete cessation of airflow) plus hypopneas, $(50 \%$ reduction in airflow associated with oxygen desaturation and/ or arousal from sleep), divided by the number of hours of recorded sleep. An AHI of 5 or greater indicates the presence of OSA, and more than 30 indicates a severe degree of OSA. Obstructive Sleep Apnoea occurs in an estimated $24 \%$ of middle-aged males and $9 \%$ of middle-aged females as defined by an $\mathrm{AHI}>5$ on sleep study.

\section{Pathophysiology:}

Daytime sleepiness is a characteristic feature of OSA and can be assessed using the Epworth Sleepiness Scale (ESS). There are 8 questions in the scale, which ask a patient how likely they are to doze off in certain situations. A score greater than 10 out of a possible 24 , indicates subjective daytime sleepiness. Obstructive Sleep Apnoea occurs as a result of collapse of the upper airway. The upper airway between the back of the nose and the top of the larynx is supported only by muscle tone. With sleep onset, this muscle tone is reduced and the airway narrows. The airspeed through this narrowed upper airway increases and results in vibration of the upper airway, with snoring. The airway may become so narrow that it collapses, usually on inspiration, resulting in an Apnoea or hypopnea. Since air can no longer enter the lungs, the oxygen level in the blood falls, and carbon dioxide levels rise. These changes are sensed by receptors in the carotid artery in the neck and also in the brain, and stimulate increased respiratory effort via the abdominal and chest wall muscles. ${ }^{12,13}$. These efforts are futile against the occluded upper airway. The fall in oxygen levels, the rise in carbon dioxide levels and increased respiratory effort may precipitate an arousal from sleep. Muscle tone then returns to the upper airway which allows breathing to recommence. Surges of sympathetic nerve activity occur during the period of apnea which may contribute to the cardiovascular consequences of OSA. The duration of this obstruction may last up to a minute and occasionally longer, and may occur hundreds of times during the night. The repeated fragmentation results in poor sleep quality and excessive daytime sleepiness.

Symptoms and Complications of OSA Repeated fragmentation of sleep from OSA results in poor quality of sleep, and symptoms of sleep deprivation. Apart from daytime sleepiness, which does not necessarily relate to the severity of the OSA, there are a wide range of health concerns. Cognitive function can be significantly impaired resulting in difficulty concentrating, depression, learning and memory difficulties, personality changes, and hyperactivity in children. Morning headaches, decreased libido and impotence in men can result from OSA, which can also increase driving risk and impair work performance. OSA has been 
linked to several cardiovascular complications. OSA is an independent risk factor for the development of hypertension and insulin resistance, and is associated with heart attack, cardiac arrhythmia, and stroke. Bed partners are also significantly affected, with their own sleep being disrupted, even as they sleep, resulting in symptoms of sleep deprivation. Licensing authorities have strict guidelines on how sleepiness and driving risk should be addressed.

Risk Factors for OSA: There are a number of recognized risk factors for OSA. These include: Male gender, increasing age, Body Mass Index $>30$, Neck circumference $>42$ $\mathrm{cm}$, enlarged tonsils, Alcohol, Smoking, Post-menopause, Sleeping tablets.

Investigation of OSA: In view of the high prevalence of OSA, and the potentially serious consequences of untreated OSA, investigation is essential in order to determine the most effective treatment. Investigation should be considered if the patient presents with persistent snoring, and at least one other associated symptom. Most commonly this will be excessive daytime sleepiness, but may include any of the symptoms mentioned above. Investigations can be divided into four categories: Level 1Full monitored overnight polysomnography undertaken in a specialist unit with overnight observation. Involving both respiratory, limb and EEG leads. Level 2.- Full overnight polysomnography, without overnight observation. This might be in a specialist unit, or at home. Level 3-Monitoring of respiratory parameters and oximetry with pulse rate, and usually undertaken in the home. Level 4Pulse oximetry, measuring oxygen desaturation and pulse rate only. Usually undertaken in the home. Level 1 studies have always been considered to be the 'gold standard' investigation for sleep disorders.
Treatments of OSA:

There are of four broad categories of effective treatments for OSA.

1. Lifestyle. Lifestyle changes can be very effective in mitigating the symptoms of sleep apnea.

Weight loss is most important in all those who are overweight. However approximately $25 \%$ of those suffering from OSA are not overweight but may exhibit some craniofacial characteristics such as micro- or retrognathia. Alcohol in the evening should be reduced to $<2$ units. Both alcohol and sleeping tablets relax the pharyngeal muscles allowing the pharyngeal walls to collapse more easily.

Smoking results in irritation and swelling of the pharyngeal space, increasing the likelihood of snoring and OSA. Snoring and OSA is almost always worse when lying on the back. Sewing a pocket into the back of the pajamas to hold a tennis ball or golf ball will ensure that lying on the back is impossible.

2. Continuous Positive Air Pressure (CPAP). This is the "gold standard" treatment for OSA. Delivered via a nasal or oronasal mask, pressurized air is used to splint open the floppy upper airway. It is a cumbersome, but extremely effective therapy. As many as $70 \%$ of patients can tolerate therapy long term. CPAP can improve quality of life, cognitive function, reduce driving risk, hypertension and cardiovascular risk, particularly in symptomatic patients with severe disease.

3. Mandibular Advancement Splints (MAS). This works by holding the mandible forward. Since the tongue is attached to the mandible, the tongue is held forward preventing it from falling backwards and 
causing obstruction. There are a number of devices which are generally effective for snoring, mild and even moderate OSA. They are most effective in patients with mild OSA which is worst in the supine position in non-obese patients. Patients who may be suitable for treatment with a MAS should be referred to a dentist who has an interest in this area. It is very important that the device is properly manufactured and customized for each patient. A number of cheaper appliances are available for purchase over the counter or from the internet. These cannot be recommended.

4. Surgery. Whilst this used to be a very popular treatment for OSA, Uvulopalatopharyngoplasty (UPPP) may improve snoring and sometimes cure OSA, particularly more severe disease. Surgery can be used to correct obstructions in the airways.

This surgery removes tissue from the rear of the mouth and to $p$ of the throat. The tissuesr emoved include parts of the uvula (the flap of tissue that hangs do wn at the back of the mouth), the soft palate, and thep harynx.
Tonsils and adenoids are usually rem oved in this operation. This operation significantly improves sleep apneain slightly more than half of all cases. Tonsillectomy is most effective when the patient has large tonsils. Nasal surgery can be used to relieve obstruction. More extensive procedures are available for selected cases where craniofacial abnormalities are impinging on the upper airway.

The most common surgery is called UPPP, Uvulopalatopharngyoplasty. Reconstructive surgery is possible for those whose OSA is due to constriction of the airway by lower jaw deformities. Genioplasty, which is a procedure that plastic surgeons usually perform to reshape a patient's chin to improve his or her appearance, is now being done to reshape the upper airway in patients with OSA. When other forms of treatment are not successful, obstructive sleep apnea may be treated by tracheotomy.

\author{
S M Khorshed Alam Mazumder \\ Professor \& Head \\ Dept. of Otolaryngology \& Head Neck Surgery \\ Holy Family Red Crescent Medical College.
}

